\title{
Investigation of the fine structure of antihydrogen
}

https://doi.org/10.1038/s41586-020-2006-5

Received: 7 December 2018

Accepted: 20 December 2019

Published online: 19 February 2020

\section{Open access}

Check for updates

\section{The ALPHA Collaboration*}

At the historic Shelter Island Conference on the Foundations of Quantum Mechanics in 1947, Willis Lamb reported an unexpected feature in the fine structure of atomic hydrogen: a separation of the $2 \mathrm{~S}_{1 / 2}$ and $2 \mathrm{P}_{1 / 2}$ states ${ }^{1}$. The observation of this separation, now known as the Lamb shift, marked an important event in the evolution of modern physics, inspiring others to develop the theory of quantum electrodynamics ${ }^{2-5}$. Quantum electrodynamics also describes antimatter, but it has only recently become possible to synthesize and trap atomic antimatter to probe its structure. Mirroring the historical development of quantum atomic physics in the twentieth century, modern measurements on anti-atoms represent a unique approach for testing quantum electrodynamics and the foundational symmetries of the standard model. Here we report measurements of the fine structure in the $n=2$ states of antihydrogen, the antimatter counterpart of the hydrogen atom. Using optical excitation of the 1S-2P Lyman- $\alpha$ transitions in antihydrogen ${ }^{6}$, we determine their frequencies in a magnetic field of 1 tesla to a precision of 16 parts per billion. Assuming the standard Zeeman and hyperfine interactions, we infer the zero-field fine-structure splitting $\left(2 \mathrm{P}_{1 / 2}-2 \mathrm{P}_{3 / 2}\right)$ in antihydrogen. The resulting value is consistent with the predictions of quantum electrodynamics to a precision of 2 per cent. Using our previously measured value of the $1 \mathrm{~S}-2 \mathrm{~S}$ transition frequency ${ }^{6,7}$, we find that the classic Lamb shift in antihydrogen $\left(2 \mathrm{~S}_{1 / 2}-2 \mathrm{P}_{1 / 2}\right.$ splitting at zero field) is consistent with theory at a level of 11 per cent. Our observations represent an important step towards precision measurements of the fine structure and the Lamb shift in the antihydrogen spectrum as tests of the chargeparity-time symmetry ${ }^{8}$ and towards the determination of other fundamental quantities, such as the antiproton charge radius ${ }^{9,10}$, in this antimatter system.
The fine-structure splitting of the $n=2$ states of hydrogen is the separation of the $2 \mathrm{P}_{3 / 2}$ and $2 \mathrm{P}_{1 / 2}$ levels at zero magnetic field. This splitting, predicted by the Dirac theory of relativistic quantum mechanics ${ }^{11}$, originates from the spin-orbit interaction between the non-zero orbital angular momentum $(L=1)$ and the electron spin. The 'classic' Lamb shift is defined as the splitting between the $2 \mathrm{~S}_{1 / 2}$ and $2 \mathrm{P}_{1 / 2}$ states at zero field ${ }^{12}$, and is a manifestation of the interaction of the electron with the quantum fluctuations of the vacuum electromagnetic field, an effect explained by quantum electrodynamics (QED) $)^{12-14}$. Today, it is understood that the classic Lamb shift in hydrogen is dominated by the QED effects on the $2 \mathrm{~S}$ energy level, and that the $1 \mathrm{~S}$ level receives even stronger QED corrections than the $2 S$ level $^{12,13}$. Although QED corrections in levels $n \neq 2$ are now also sometimes referred to as Lamb shifts, in this Article we restrict our definition of the Lamb shift to be the classic $n=2$ shift.

In a magnetic field, the Zeeman effect causes the $2 \mathrm{P}_{3 / 2}$ state to also split into four sublevels (labelled $2 \mathrm{P}_{\mathrm{a}}, 2 \mathrm{P}_{\mathrm{b}}, 2 \mathrm{P}_{\mathrm{c}}$ and $2 \mathrm{P}_{\mathrm{d}}$ ), whereas the $2 \mathrm{~S}_{1 / 2}$ and $2 \mathrm{P}_{1 / 2}$ states each split into two $\left(2 \mathrm{~S}_{\mathrm{ab}}\right.$ and $2 \mathrm{~S}_{\mathrm{cd}} ; 2 \mathrm{P}_{\mathrm{e}}$ and $\left.2 \mathrm{P}_{\mathrm{f}}\right)$. These fine-structure levels further split into two hyperfine states owing to the proton spin (see Fig. 1 for the expected energy levels for the case of antihydrogen, where the spin orientations are reversed with respect to those of hydrogen.)
Lamb's original work used the then newly developed techniques of an excited-state atomic hydrogen beam and resonant microwave spectroscopy to study direct transitions between the $n=2$ fine-structure states in various magnetic fields. The Lamb shift was then determined to $10 \%$ precision by extrapolating frequency measurements to zero field ${ }^{1}$. Here, we report the observation of the splitting between the $2 \mathrm{P}_{c}$ and $2 \mathrm{P}_{\mathrm{f}}$ states in antihydrogen in a field of $1 \mathrm{~T}$, by studying laser-induced transitions from the ground state. Assuming the validity of the Zeeman and hyperfine interactions, and using the value of the previously measured $1 S-2 S$ transition frequency ${ }^{7}$, we infer from our results the values of the zero-field fine-structure splitting and the classic Lamb shift in antihydrogen. Such studies have become possible owing to the combination of several recent advances: the accumulation ${ }^{15}$ of hundreds of anti-atoms in each run, their confinement for many hours ${ }^{16}$, control of the hyperfine polarization of the antihydrogen samples ${ }^{17}$ and the development of a narrow-line, pulsed, Lyman- $\alpha$ laser ${ }^{6,18}$.

Details of the production, trapping and control of antihydrogen in the ALPHA experiment have been provided elsewhere ${ }^{6,7,15-25}$, so the following description is brief. The ALPHA-2 apparatus (Fig. 2) incorporates a cylindrical magnetic trapping volume (about $400 \mathrm{~cm}^{3}$ ) for neutral anti-atoms; the magnetic-field minimum at the centre of the trap was 


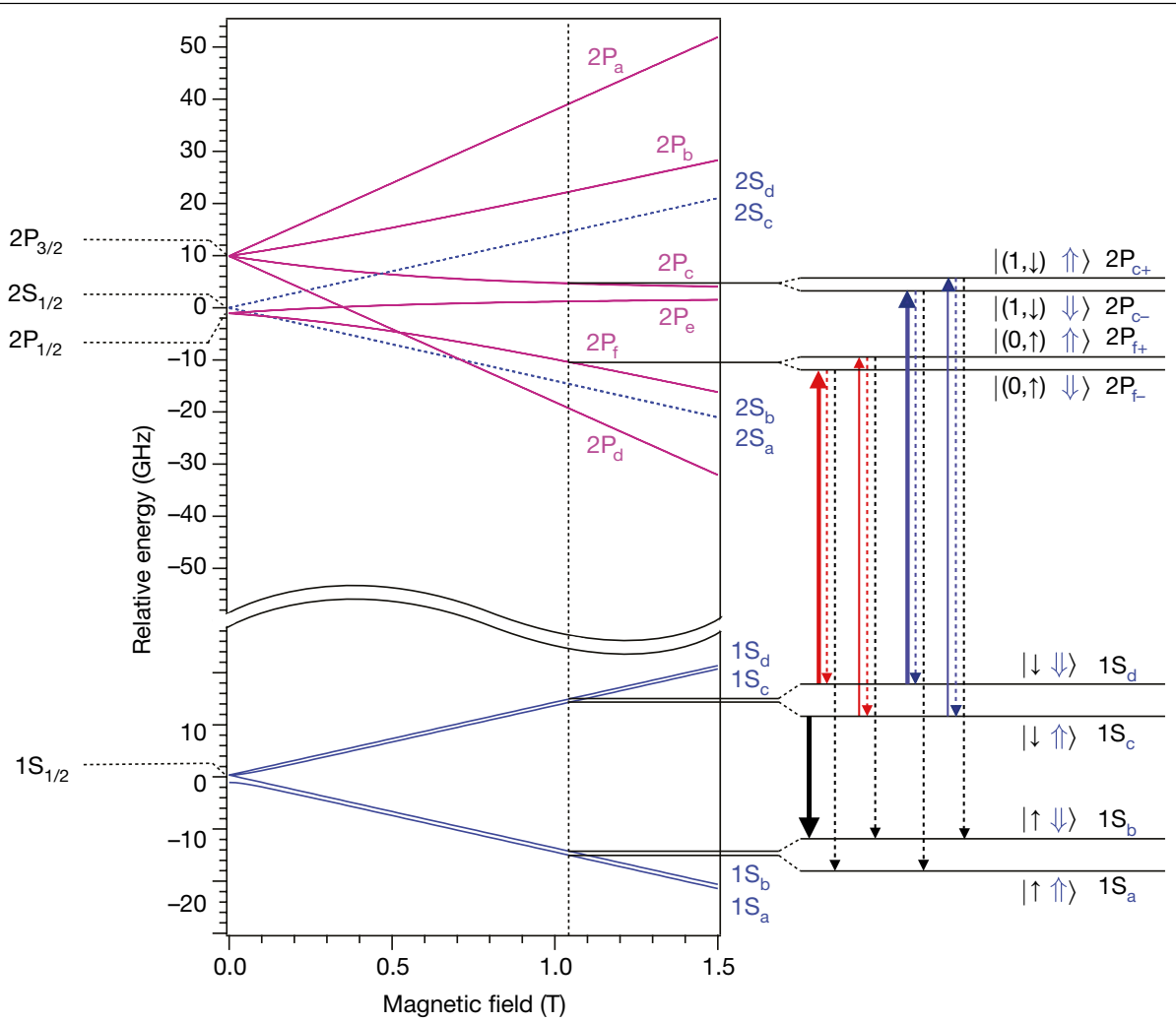

Fig. 1 | Expected antihydrogen energy levels. Calculated energies of the fine structure and the hyperfine sublevels of the $1 \mathrm{~S}_{1 / 2}, 2 \mathrm{~S}_{1 / 2}, 2 \mathrm{P}_{3 / 2}$ and $2 \mathrm{P}_{1 / 2}$ states are shown as functions of magnetic-field strength. The spin orientations for antihydrogen are shown; they are reversed for hydrogen. The centroid energy difference, $E_{1 \mathrm{~S}-2 \mathrm{~S}}=2.4661 \times 10^{15} \mathrm{~Hz}$, has been suppressed on the vertical axis. Details of the energy levels relevant to this work at a magnetic field of $B=1.0329 \mathrm{~T}$ are shown on the right. Each state is labelled using conventional notation. For the $1 \mathrm{~S}$ and $2 \mathrm{~S}$ states, the hyperfine states are labelled with subscripts a-d in order of increasing energy (see, for example, ref. ${ }^{7}$ ); namely, $\mathrm{S}_{\mathrm{a}}=|\uparrow \Uparrow\rangle, \mathrm{S}_{\mathrm{b}}=|\uparrow \downarrow\rangle, \mathrm{S}_{\mathrm{c}}=|\downarrow \Uparrow\rangle$ and $\mathrm{S}_{\mathrm{d}}=|\downarrow \downarrow\rangle$, where the ket notation represents the positron spin (left; $\downarrow$ or $\uparrow$ ) and antiproton spin (right; $\Downarrow$ or $\Uparrow$ ) states in the highfield limit. The labels $\mathrm{S}_{\mathrm{ab}}$ and $\mathrm{S}_{\mathrm{cd}}$ are used when the antiproton spins are unpolarized. For the 2P states, the fine-structure splittings are labelled with

set to $1.0329 \pm 0.0004 \mathrm{~T}$ for this work. (All uncertainties given herein are $1 \sigma$.) By combining 90,000 trapped antiprotons from the CERN Antiproton Decelerator ${ }^{23}$ and three million positrons from a positron accumulator $^{24,25}$, about $10-30$ cold (below $0.54 \mathrm{~K}$ ) anti-atoms are confined in the magnetic trap in a 4-min cycle. Under normal conditions, the storage lifetime ${ }^{16}$ of the trapped antihydrogen is greater than $60 \mathrm{~h}$, which permits loading from repeated cycles $^{15}$ to obtain hundreds of antihydrogen atoms in a few hours.

Two types of antihydrogen samples were used in these studies. The positron spin of an antihydrogen atom confined in the ALPHA-2 trap is necessarily polarized, because only the $1 S_{c}$ and $1 S_{d}$ states can be magnetically trapped (Fig. 1). The antiproton spin, on the other hand, is unpolarized a priori, with both orientations equally likely. Thus, the initial samples are singly spin-polarized. On the other hand, doubly spin-polarized samples, in which both the positron and antiproton spins are polarized, can be prepared by injecting microwaves to resonantly drive the $1 S_{c}$ atoms to the untrappable $1 S_{b}$ state (Fig. 1), effectively depopulating the $1 \mathrm{~S}_{\mathrm{c}}$ state from the trap ${ }^{17}$.

Spectroscopy in the vacuum ultraviolet range is challenging even for ordinary atoms, owing in part to the lack of convenient laser sources and optical components ${ }^{26-28}$. Our pulsed, coherent 121.6-nm radiation was produced by generating the third harmonic of $365-\mathrm{nm}$ pulses in a $\mathrm{Kr} / \mathrm{Ar}$ gas mixture at a repetition rate of $10 \mathrm{~Hz}\left(\right.$ ref. $\left.^{18}\right)$. The typical pulse width subscripts a-f in order of decreasing energy at low magnetic fields, whereas the hyperfine splitting due to the antiproton spin is specified by subscripts + and for spin parallel $(\Uparrow)$ and anti-parallel $(\Downarrow)$ to the magnetic field in the high-field limit, respectively. The symbol $(\downarrow, \uparrow)$ in the figure indicates that the positron spin states are mixed for the $2 \mathrm{P}_{\mathrm{c}}$ and $2 \mathrm{P}_{\mathrm{f}}$ states. The vertical solid arrows indicate the one-photon laser transitions probed here: $1 \mathrm{~S}_{d} \rightarrow 2 \mathrm{P}_{\mathrm{f}-}$ (bold red), $1 \mathrm{~S}_{\mathrm{c}} \rightarrow 2 \mathrm{P}_{\mathrm{f}^{+}}$(thin red), $1 \mathrm{~S}_{\mathrm{d}} \rightarrow 2 \mathrm{P}_{\mathrm{c}^{-}}$(bold blue) and $1 \mathrm{~S}_{\mathrm{c}} \rightarrow 2 \mathrm{P}_{\mathrm{c}^{+}}$(thin blue). The dashed red and blue arrows indicate relaxation to the same trappable level, which is not detectable in the present experiment, and the dashed black arrows indicate relaxation to untrappable levels, which is detectable via annihilation signals (see text). The bold black arrow shows the microwave transition used to eliminate $1 \mathrm{~S}_{\mathrm{c}}$ state atoms to prepare a doubly spin-polarized antihydrogen sample.

at $121.6 \mathrm{~nm}$ was $12 \mathrm{~ns}$, and the bandwidth was estimated from the Fourier transform of the temporal pulse shape to be $65 \mathrm{MHz}$ (full-width at halfmaximum, FWHM). The 121.6-nm light was linearly polarized because of the three-photon mixing of linearly polarized 365-nm light. In the antihydrogen trap, the polarization vector was nearly perpendicular to the direction of the axial magnetic field. The laser beam had a radius of $3.6 \mathrm{~mm}$ and was roughly collimated across the trapping region (Fig. 2). The average pulse energies in the antihydrogen trapping volume ranged from $0.44 \mathrm{~nJ}$ to $0.72 \mathrm{~nJ}$ over different runs, as evaluated from the pulse waveforms recorded with a calibrated, solar-blind photomultiplier detector.

In this experiment, single-photon transitions from the $1 S_{c}\left(1 S_{d}\right)$ states to the $2 \mathrm{P}_{\mathrm{c}^{+}}\left(2 \mathrm{P}_{\mathrm{c}^{-}}\right)$and $2 \mathrm{P}_{\mathrm{f}_{+}}\left(2 \mathrm{P}_{\mathrm{f}_{-}}\right)$states are driven by the 121.6-nm light (red and blue arrows in Fig. 1). When antihydrogen is excited to the $2 \mathrm{P}_{\mathrm{c} \pm}$ or $2 \mathrm{P}_{\mathrm{ft}}$ state, it decays to the ground-state manifold within a few nanoseconds by emitting a photon at $121.6 \mathrm{~nm}$. The mixed nature of the positron spin states in the $2 \mathrm{P}_{\mathrm{c}^{+}}\left(2 \mathrm{P}_{\mathrm{c}-}\right)$ and $2 \mathrm{P}_{\mathrm{f}^{+}}\left(2 \mathrm{P}_{\mathrm{f}-}\right)$ states implies that these states can decay to the $1 \mathrm{~S}_{\mathrm{b}}\left(1 \mathrm{~S}_{\mathrm{a}}\right.$ ) states via a positron spin flip (black dashed arrows in Fig. 1). Atoms in these final states are expelled from the trap and are annihilated on the trap walls. Annihilation products (charged pions) are in turn detected by a silicon vertex detector ${ }^{29}$ with an efficiency greater than $80 \%$.

Table 1 summarizes our data. In total, four series of measurements were performed using either singly or doubly spin-polarized samples. 


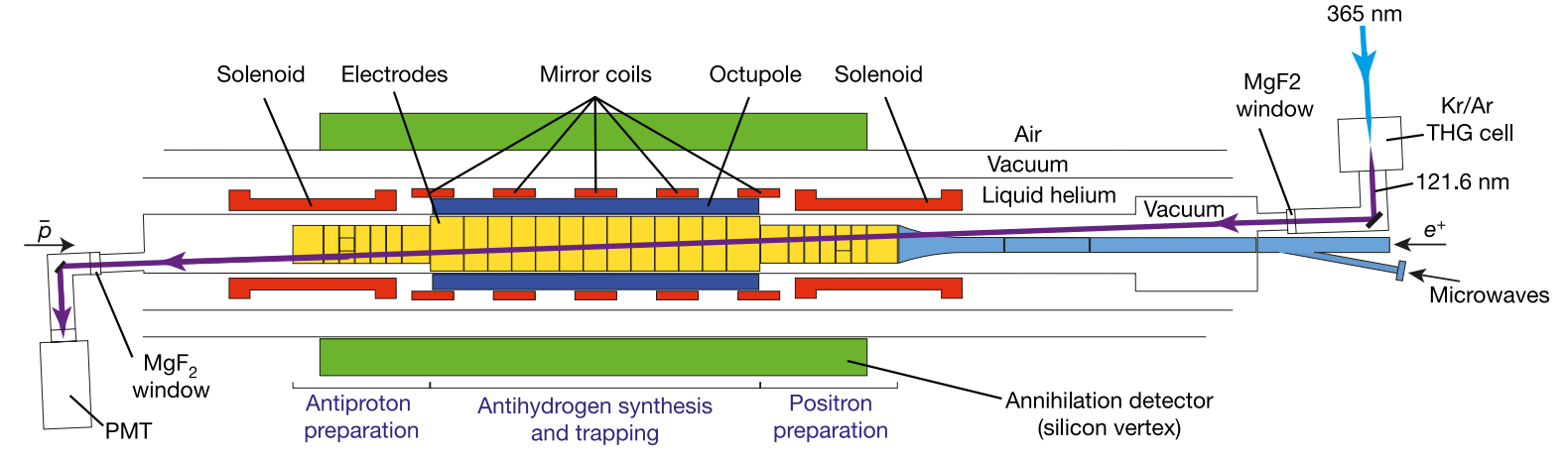

Fig. 2 The ALPHA-2 central apparatus. A cylindrical trapping volume for neutral antimatter with a diameter of $44.35 \mathrm{~mm}$ and an axial length of $280 \mathrm{~mm}$ is located inside several Penning trap electrodes and surrounded by an octupole coil, five mirror coils and two solenoids, all superconducting. The three-layer silicon vertex annihilation detector is shown schematically in green. Laser light (purple line) enters from the positron $\left(e^{+}\right)$side (right) and is transmitted to the antiproton $(\bar{p})$ side (left) through vacuum-ultraviolet-grade
$\mathrm{MgF}_{2}$ ultrahigh-vacuum windows. The laser beam crosses the trap axis at an angle of $2.3^{\circ}$. The transmitted $121.6-\mathrm{nm}$ pulses are detected by a solar-blind photomultiplier tube (PMT) at the antiproton side. Microwaves used to prepare the doubly spin-polarized samples are introduced from the positron side through a waveguide, shown in blue. The external solenoid magnet for the Penning traps is not shown here. THG, third-harmonic generation.
The Series 1 data, previously reported in ref. $^{6}$, have been reanalysed. Each series consisted of two or four runs, and in each run about 500 antihydrogen atoms were accumulated over approximately two hours, typically involving over 30 production cycles. The trapped anti-atoms were then irradiated for about two hours by a total of 72,000 laser pulses at twelve different frequencies (that is, 6,000 pulses per frequency point for each run) spanning the range $-3.10 \mathrm{GHz}$ to $+2.12 \mathrm{GHz}$ relative to the expected (hydrogen) transition frequencies. The laser frequency was changed every $20 \mathrm{~s}$ in a non-monotonic fashion to minimize effects related to the depletion of the sample of antihydrogen. After the laser exposure, the remaining antihydrogen atoms were released by shutting down the trap magnets, typically in $15 \mathrm{~s}$, and counted via detection of their annihilation events. $40-60 \%$ of the trapped antihydrogen atoms experienced resonant, laser-induced spin flips, and their annihilations were detected during the two-hour laser irradiation period.

A combination of time-gated antihydrogen detection (enabled by the use of a pulsed laser), the accumulation of a large number of antiatoms and the use of supervised machine-learning analysis ${ }^{29}$ (based on a boosted decision-tree classifier) suppressed the background to a negligible level (less than 2 counts per 2 -h irradiation period).

The measured spectra, obtained from counting the laser-induced spin-flip events, are shown in Fig. 3 for both singly and doubly spinpolarized antihydrogen samples. For each run, the probability at each frequency point is determined from dividing the number of annihilation events recorded at that frequency by the total number of trapped atoms in that run, and further dividing by the ratio of the average laser energy to a standard value of $0.5 \mathrm{~nJ}$. The normalization to the standard laser energy is to account for the expected linear dependence of the transition probability on the laser power in our regime. The data plotted in Fig. 3 are spectrum-averaged over the runs for each series. For the singly polarized sample (Fig. 3a), each transition shows a linewidth of about $1.5 \mathrm{GHz}$ (FWHM). This is consistent with the expected Doppler broadening in our trapping condition ( $1 \mathrm{GHz}$ FWHM) and the hyperfine splitting of the $1 \mathrm{~S}-2 \mathrm{P}_{\mathrm{f}}$ and $1 \mathrm{~S}-2 \mathrm{P}_{\mathrm{c}}$ transitions $(0.71 \mathrm{GHz}$ for both transitions). The hyperfine structure cannot be resolved in these singly polarized samples owing to the Doppler broadening.

Figure $3 b$ shows the spectra obtained from doubly spin-polarized antihydrogen samples. For these data, microwave radiation of $28 \mathrm{GHz}$ (power $0.4 \mathrm{~W}$, measured at the trap entrance) was applied before the start of optical spectroscopy, in the form of a 9-MHz sweep, covering the $1 \mathrm{~S}_{\mathrm{c}}-1 \mathrm{~S}_{\mathrm{b}}$ transition in the magnetic-field minimum ${ }^{17}$. As shown in Table 1, about half of the total trapped antihydrogen atoms underwent a positron spin-flip and annihilated during microwave irradiation. This is consistent with our experience from earlier studies, in which $1 \mathrm{~S}_{\mathrm{c}}$ state atoms were removed with about $95 \%$ efficiency ${ }^{7,17}$. The spectral lines of the 1S-2P transitions in doubly spin-polarized antihydrogen (Fig. 3b) are narrower than those in the singly spin-polarized samples (Fig. 3a) because the former involves only one hyperfine state in the ground state. The peaks are red-shifted because the frequencies of the transition from the $1 S_{d}$ state to the $2 P_{f}$ and $2 P_{c}$ states are expected to be about $700 \mathrm{MHz}$ lower than those from the $1 \mathrm{~S}_{\mathrm{c}}$ state. The observed width of $\sim 1 \mathrm{GHz}$ FWHM of these lines is in agreement with the Doppler width expected for our trapping conditions.

The procedure used to extract the frequencies of the fine-structure transitions and to evaluate their associated uncertainties is described in Methods. We summarize the results of this analysis in Table 2. A simulation was used to model the motion of trapped antihydrogen atoms in the ALPHA-2 trap and their interaction with pulsed laser radiation. The resonance transition frequencies were obtained by comparing simulated and experimental lineshapes. Extensive investigations were performed to evaluate systematic uncertainties in our measurement (Table 3). The validity of our analysis procedure was tested by using

\section{Table 1 | Experimental parameters and number of detected events}

\begin{tabular}{|c|c|c|c|c|c|c|c|c|c|c|}
\hline Series & $\begin{array}{l}\text { Sample } \\
\text { polarization }\end{array}$ & $\begin{array}{l}\text { Transition } \\
\text { probed }\end{array}$ & $\begin{array}{l}\text { Number } \\
\text { of runs }\end{array}$ & $\begin{array}{l}\text { Average pulse } \\
\text { energy (pJ) }\end{array}$ & $\begin{array}{l}\text { Number of } \\
\text { frequencies }\end{array}$ & $\begin{array}{l}\text { Number of pulses } \\
\text { per frequency }\end{array}$ & $\begin{array}{l}\text { Number of } \\
\text { trapped atoms }\end{array}$ & $\begin{array}{l}\text { Microwave } \\
\text { counts }\end{array}$ & $\begin{array}{l}\text { Laser } \\
\text { counts }\end{array}$ & $\begin{array}{l}\text { Counts upon } \\
\text { release }\end{array}$ \\
\hline 1 & Single & $1 \mathrm{~S}_{\mathrm{cd}} \rightarrow 2 \mathrm{P}_{\mathrm{c} \pm}$ & 4 & 600 & 12 & 24,000 & 2,004 & - & 1,197 & 807 \\
\hline 2 & Single & $1 \mathrm{~S}_{\mathrm{cd}} \rightarrow 2 \mathrm{P}_{\mathrm{f} \pm}$ & 4 & 550 & 12 & 24,000 & 2,012 & - & 1,075 & 937 \\
\hline 3 & Double & $1 \mathrm{~S}_{\mathrm{d}} \rightarrow 2 \mathrm{P}_{\mathrm{c}^{-}}$ & 2 & 440 & 12 & 12,000 & 1,044 & 527 & 229 & 288 \\
\hline 4 & Double & $1 S_{d} \rightarrow 2 P_{f-}$ & 2 & 720 & 12 & 12,000 & 971 & 463 & 341 & 167 \\
\hline
\end{tabular}

The experimental parameters, together with the number of antihydrogen events detected during the microwave irradiation, the laser irradiation and the release of the remaining atoms, are tabulated for each series. The machine-learning analysis identifies annihilation events with an estimated efficiency of 0.849 for the microwave irradiation, 0.807 for the laser irradiation and 0.851 for the release of the remaining atoms. The number of counts is corrected for the detection efficiencies. The number of trapped atoms is derived from the sum of the other counts. 

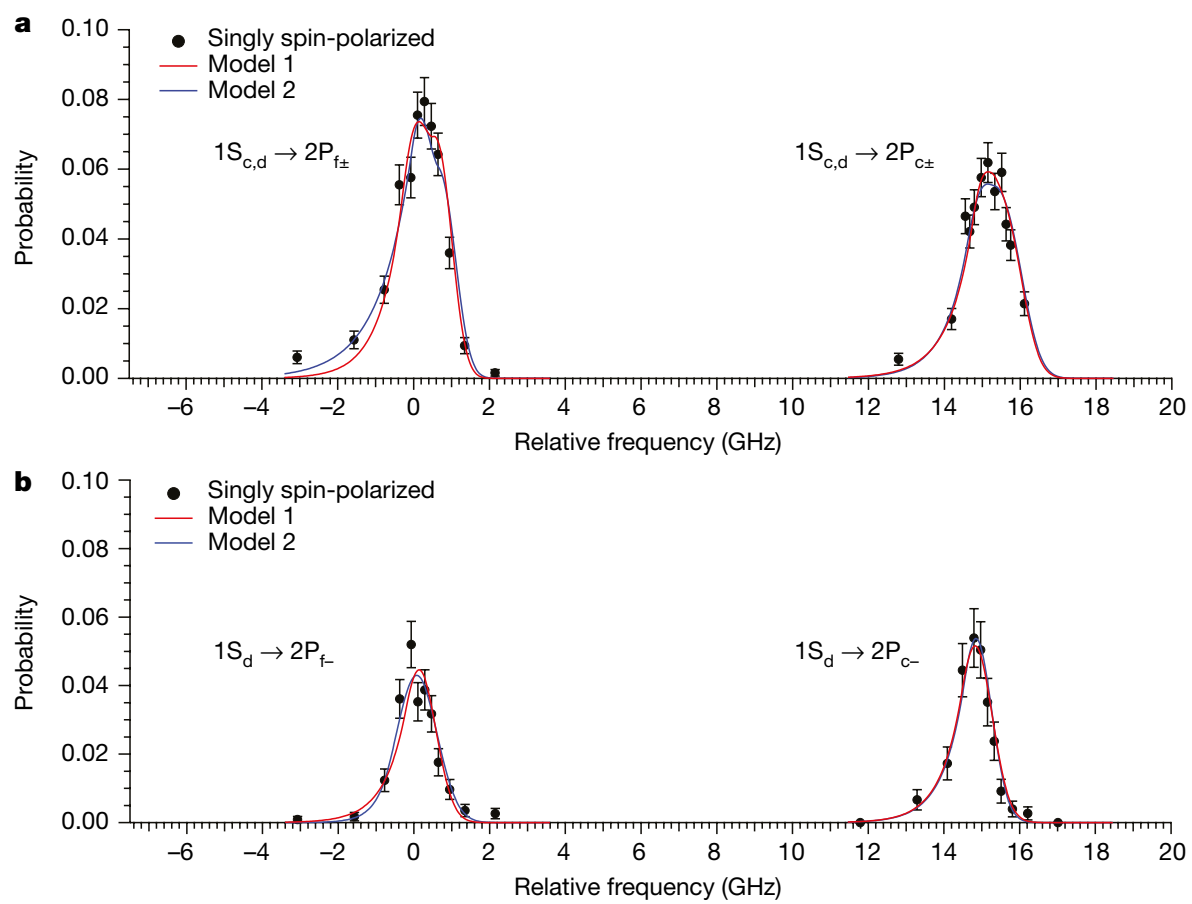

Fig. 3 | 1S-2P fine-structure spectrum of antihydrogen. a, b, Experimental data (filled circles) and fitted lineshapes for singly spin-polarized (a) and doubly spin-polarized (b) antihydrogen samples. The data points were obtained from the detected spin-flip events, normalized to the total number of trapped antihydrogen atoms, for a laser pulse energy of $0.5 \mathrm{~nJ}$. The error bars are $1 \sigma$ counting uncertainties. The frequency is offset by $2,466,036.3 \mathrm{GHz}$. We note that no data were taken between the two peaks $(-2-12 \mathrm{GHz})$. The red fit curves were obtained via our standard fitting procedure (Model1), and the blue curves were derived from an alternative fitting model (Model 2), illustrating the sensitivity of our results to the fitting procedure. See text and Methods for detailed discussion. different lineshape-fitting models. Two representative curve fits are shown in Fig. 3. The fit of Model 1 uses a function constrained to fit the simulation shape, whereas in Model 2 the shape parameters of this function are allowed to vary to best fit the experimental data; see Methods for details. The sensitivity of the results to the experimental and simulation parameters was tested by repeating the analysis procedure for a number of simulations with varied input. These included the initial antihydrogen conditions (such as the initial temperature, the quantum state, and the cloud diameter of antihydrogen at formation) and laser properties (such as linewidth, beam waist size and beam position); see Methods and Extended Data Fig. 1. Other sources of systematic uncertainties include the calibration accuracy and a possible frequency drift of the wavemeter, frequency shifts of the 730-nm amplification laser cavity, and possible incomplete clearing of the $1 S_{c}$ state in the preparation of the doubly spin-polarized samples (Table 3 and Methods).

Within the uncertainties, the measured transition frequencies agree with theoretical expectations for hydrogen for all four series (Table 2, Fig. 4). The fact that the four measurements are consistent, despite having different systematics, increases the confidence in our overall results. The results can be combined to give a test of charge-parity-time (CPT) invariance in the $1 \mathrm{~S}-2 \mathrm{P}$ transitions at the level of 16 parts per billion (Fig. 4).

Fundamental physical quantities of antihydrogen can be extracted from our optical measurements of the 1S-2P transitions by combining them with our earlier measurement of the $1 S-2 S$ transition in the same magnetic trapping field ${ }^{7}$. From the weighted average of the results between the singly polarized and doubly polarized measurements (Table 1), we obtain a $2 \mathrm{P}_{\mathrm{c}^{-}}-2 \mathrm{P}_{\mathrm{f}-}$ splitting of $14.945 \pm 0.075 \mathrm{GHz}$, a $2 \mathrm{~S}_{\mathrm{d}}-2 \mathrm{P}_{\mathrm{c}-}$ splitting of $9.832 \pm 0.049 \mathrm{GHz}$ and a $2 \mathrm{~S}_{\mathrm{d}}-2 \mathrm{P}_{\mathrm{f}-}$ splitting of $24.778 \pm 0.060 \mathrm{GHz}$ at $1.0329 \mathrm{~T}$ (Methods). Only two of these three splittings are independent, and they all agree with the values predicted for hydrogen in the same field.

In interpreting our data, we categorize features in the spectrum based on the order of the fine-structure constant $\alpha$ in a perturbative series expansion in quantum field theory (which is assumed to be valid for the purpose of our categorization). Those features that can be described by the Dirac theory (the Zeeman, hyperfine and fine-structure effects) are referred to as 'tree-level effects' and follow from the lower-order terms (up to order $\sim \alpha^{2} \mathrm{Ry}$, where Ry is the Rydberg constant). On the other hand, the Lamb shift originates from the so-called 'loop effects' (order $\sim \alpha^{3} \mathrm{Ry}$ ), the calculation of which requires the concept of renormalization

Table 2 | 1S-2P transition frequencies

\begin{tabular}{|c|c|c|c|c|}
\hline & Sample spin polarization & Antihydrogen $f_{\text {res }}(\exp )(\mathrm{MHz})$ & Hydrogen $f_{\text {res }}($ th) $(\mathrm{MHz})$ & Difference $f_{\text {res }}(\exp )-f_{\text {res }}($ th $)(\mathrm{MHz})$ \\
\hline $1 \mathrm{~S}_{\mathrm{cd}} \rightarrow 2 \mathrm{P}_{\mathrm{ct}}$ & Single & $2,466,051,659(62)$ & $2,466,051,625$ & 34 \\
\hline $1 \mathrm{~S}_{\mathrm{cd}} \rightarrow 2 \mathrm{P}_{\mathrm{f \pm}}$ & Single & $2,466,036,611(88)$ & $2,466,036,642$ & -31 \\
\hline $1 \mathrm{~S}_{\mathrm{d}} \rightarrow 2 \mathrm{P}_{\mathrm{c}^{-}}$ & Double & $2,466,051,189(76)$ & $2,466,051,270$ & -81 \\
\hline $1 S_{d} \rightarrow 2 P_{f-}$ & Double & $2,466,036,395(81)$ & $2,466,036,287$ & 108 \\
\hline
\end{tabular}

The experimentally determined transition frequencies for antihydrogen $f_{\text {res }}$ (exp) (with $1 \sigma$ errors in parentheses) are compared with the theoretically expected values for hydrogen $f_{\text {res }}($ th) at a magnetic field of $1.0329 \mathrm{~T}$. For the singly spin-polarized data, the centroid of the hyperfine states is given. The transition frequencies for hydrogen were calculated to a precision better than $1 \mathrm{MHz}$ (Methods). 
Table 3 | Summary of uncertainties

\begin{tabular}{|c|c|c|c|c|}
\hline Source of uncertainty & $\begin{array}{l}1 \mathrm{~S}_{\mathrm{d}} \rightarrow 2 \mathrm{P}_{\mathrm{c}} \text { - Doubly } \\
\text { spin-polarized (MHz) }\end{array}$ & $\begin{array}{l}1 \mathrm{~S}_{\mathrm{d}} \rightarrow 2 \mathrm{P}_{\mathrm{ff}} \text { Doubly } \\
\text { spin-polarized (MHz) }\end{array}$ & $\begin{array}{l}\mathbf{1 S}_{\mathrm{cd}} \rightarrow 2 \mathrm{P}_{\mathrm{ct}} \text { Singly } \\
\text { spin-polarized (MHz) }\end{array}$ & $\begin{array}{l}1 \mathrm{~S}_{\mathrm{cd}} \rightarrow 2 \mathrm{P}_{\mathrm{ft}} \text { Singly } \\
\text { spin-polarized }(\mathrm{MHz})\end{array}$ \\
\hline Lineshape fit statistics & 55 & 54 & 45 & 47 \\
\hline Fitting-model dependence & 24 & 42 & 17 & 62 \\
\hline Wavemeter drift & 30 & 30 & 30 & 30 \\
\hline Wavemeter offset & 18 & 18 & 18 & 18 \\
\hline 730-nm cavity frequency correction & 18 & 18 & 18 & 18 \\
\hline Residual $1 \mathrm{~S}_{\mathrm{c}}$ state atoms in doubly spin-polarized sample & 23 & 16 & 0 & 0 \\
\hline Magnetic field & 5 & 8 & 5 & 8 \\
\hline Total & 76 & 81 & 62 & 88 \\
\hline
\end{tabular}

Estimated uncertainties $(1 \sigma)$ at $121.6 \mathrm{~nm}$ for each transition (Methods).

to avoid infinities ${ }^{12-14}$. Each of the measured splittings has different sensitivity to different terms. At the level of our precision, the $2 \mathrm{P}_{\mathrm{c}}-$ $2 \mathrm{P}_{\mathrm{f}}$ splitting is sensitive to the tree-level terms with negligible QED effects, whereas the $2 \mathrm{~S}-2 \mathrm{P}_{\mathrm{f}}$ and $2 \mathrm{~S}-2 \mathrm{P}_{\mathrm{c}}$ splittings are sensitive to the field-independent Lamb shift, in addition to the tree-level terms (we note that the Lamb shift is predicted to have negligible dependence on the magnetic field ${ }^{14}$ ). The agreement between our measurement and the Dirac prediction for the $2 \mathrm{P}_{\mathrm{c}-}-2 \mathrm{P}_{\mathrm{f}-}$ splitting supports the consistency of the tree-level theory in describing the Zeeman, hyperfine and fine-structure interactions in the $2 \mathrm{P}$ states of antihydrogen. If we hence assume that we can correctly account for the tree-level effects in our measurements, we can infer from our measured splittings the values of the zero-field fine-structure splitting in antihydrogen to be $10.88 \pm 0.19 \mathrm{GHz}$. By combining the current result with the much more precisely measured $1 S-2 S$ transition frequency in antihydrogen ${ }^{7}$, we obtain a classic Lamb shift of $0.99 \pm 0.11 \mathrm{GHz}$ (Methods). If we use the theoretical value of the fine-structure splitting from the Dirac prediction (rather than treat it as a parameter), we can derive a tighter constraint on the Lamb shift, $1.046 \pm 0.035 \mathrm{GHz}$.

When considering the first measurements on an exotic system such as antihydrogen, it is necessary to adopt a framework within which it is possible to compare the results to the expectations of well established models for normal matter. The choice of which effects can be assumed to be true in interpreting the data are, of necessity, somewhat arbitrary. The approach illustrated here is based on the order of perturbation in the coupling constant $\alpha$; we have assumed (lower-order) tree-level effects in order to extract (higher-order) renormalizable loop effects.

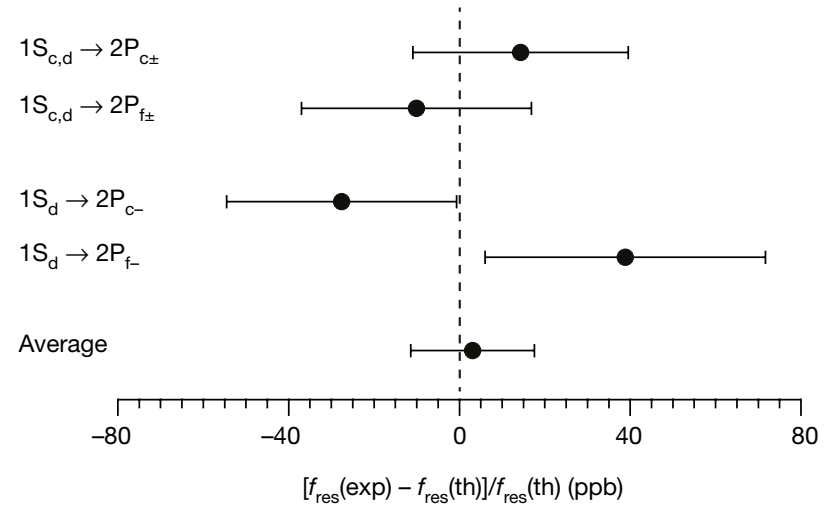

Fig. 4 | Comparison of antihydrogen and hydrogen transition frequencies. The experimentally measured frequencies for the $1 \mathrm{~S}-2 \mathrm{P}$ transitions in antihydrogen $f_{\text {res }}$ (exp) are compared with those theoretically expected for hydrogen $f_{\text {res }}($ th) (Table 2). All four measurements are consistent with hydrogen, and their average gives a combined test of CPT invariance at 16 parts per billion (ppb). The error bars are $1 \sigma$, and the calculation of the error bar for the average takes into account correlated uncertainties (Methods).
Other approaches are possible in interpreting our data. We note that if the standard theory for the hydrogen atom applies to antihydrogen, most of the expected QED effect is on the 2S level, rather than on the 2P level. Furthermore, the $1 S$ level receives approximately $n^{3}=8$ times larger QED corrections than the 2S level; hence, our earlier accurate determination of the antihydrogen $1 S-2 S$ level difference ${ }^{7}$ gives strong constraints on new interactions within the QED framework. However, it is possible that a new effect could show up in the antihydrogen classic Lamb shift while satisfying the $1 S-2 S$ constraint. See ref. ${ }^{8}$ for an example in a Lorentz-violating effective-field theory framework.

We have investigated the fine structure of the antihydrogen atom in the $n=2$ states. The splitting between the $2 \mathrm{P}_{\mathrm{c}}$ and $2 \mathrm{P}_{\mathrm{f}}$ states, two of the $2 \mathrm{P}$ Zeeman sublevels belonging to the $J=3 / 2$ and $J=1 / 2$ manifolds $(\mathrm{V}$, total angular momentum), has been observed in a magnetic field of $1 \mathrm{~T}$. The energy levels of the $1 \mathrm{~S}-2 \mathrm{P}$ transitions agree with the Dirac theory predictions for hydrogen at $1 \mathrm{~T}$ to 16 parts per billion, and their difference to $0.5 \%$. By assuming the standard Zeeman and hyperfine effects, and by combining our results with the earlier result of $1 S-2 S$ spectroscopy ${ }^{7}$, we have inferred the zero-field fine-structure splitting and the classic Lamb shift in the $n=2$ level.

These observations expand the horizons of antihydrogen studies, providing opportunities for precision measurements of the fine structure and the Lamb shift-both of which are longstanding goals in the field. Prospects exist for considerable improvements in the precision beyond this initial determination. With the advent of the ELENA ring in 2021, an upgrade to the Antiproton Decelerator with an anticipated increase in the antiproton flux, the statistical uncertainties are expected to be dramatically reduced. The development of laser cooling ${ }^{30}$ would reduce the Doppler width to a level comparable to the natural linewidth, which in turn would improve the precision of the frequency determination. It would also permit direct experimental determination of the hyperfine splitting in the $2 \mathrm{P}$ states, for which theoretical values were assumed in this study.

Such measurements will provide tests of CPT invariance that are complementary to other precision measurements in antihydrogen, such as the $1 \mathrm{~S}-2 \mathrm{~S}$ frequency and the ground-state hyperfine splitting. Furthermore, a precise value of the classic Lamb shift, combined with that of the 1S-2S interval, will permit an antimatter-only determination of the antiproton charge radius ${ }^{9,10}$, without referring to matter measurements - that is, independent of the proton charge radius puzzle ${ }^{31-33}$. These examples signify the importance of broad and complementary measurements in testing fundamental symmetries. In the absence of compelling theoretical arguments to guide the way to possible asymmetries, it is essential to address the antihydrogen spectrum as comprehensively as is practical. Finally, the results reported here demonstrate our capability to precisely and reproducibly drive vacuum ultraviolet transitions on a few antiatoms, and indicate our readiness for laser cooling of antihydrogen ${ }^{30}$, an eagerly anticipated development in antimatter studies with far-reaching implications for both spectroscopic and gravitational studies ${ }^{34}$. 


\section{Online content}

Any methods, additional references, Nature Research reporting summaries, source data, extended data, supplementary information, acknowledgements, peer review information; details of author contributions and competing interests; and statements of data and code availability are available at https://doi.org/10.1038/s41586-020-2006-5.

1. Lamb, W. E., Jr \& Retherford, R. C. Fine structure of the hydrogen atom by a microwave method. Phys. Rev. 72, 241-243 (1947).

2. Tomonaga, S. On a relativistically invariant formulation of the quantum theory of wave fields. Prog. Theor. Phys. 1, 27-42 (1946).

3. Schwinger, J. On quantum-electrodynamics and the magnetic moment of the electron. Phys. Rev. 73, 416-417 (1948).

4. Feynman, R. P. Space-time approach to quantum electrodynamics. Phys. Rev. 76 , 769-789 (1949).

5. Schweber, S. S. QED and the Men who Made it: Dyson, Feynman, Schwinger, and Tomonaga (Princeton Univ. Press, 1994).

6. Ahmadi, M. et al. Observation of the 1S-2P Lyman-a transition in antihydrogen. Nature 561, 211-215 (2018)

7. Ahmadi, M. et al. Characterization of the $1 \mathrm{~S}-2 \mathrm{~S}$ transition in antihydrogen. Nature $\mathbf{5 5 7}$ 71-75 (2018).

8. Kostelecký, V. A. \& Vargas, A. J. Lorentz and CPT tests with hydrogen, antihydrogen, and related systems. Phys. Rev. D 92, 056002 (2015).

9. Crivelli, P., Cooke, D. \& Heiss, M. W. Antiproton charge radius. Phys. Rev. D 94, 052008 (2016).

10. Eriksson, S. Precision measurements on trapped antihydrogen in the ALPHA experiment Philos. Trans. Royal Soc. A 37620170268 (2018).

11. Dirac, P. A. M. The quantum theory of the electron. Proc. R. Soc. A 117, 610-624 (1928).

12. Kinoshita, T. Quantum Electrodynamics (World Scientific, 1990).

13. Karshenboim, S. G. Precision physics of simple atoms: QED tests, nuclear structure and fundamental constants. Phys. Rep. 422, 1-63 (2005).

14. Brodsky, S. J. \& Parsons, R. G. Precise theory of the Zeeman spectrum for atomic hydrogen and deuterium and the Lamb shift. Phys. Rev. 163, 134-146 (1967).

15. Ahmadi, M. et al. Antihydrogen accumulation for fundamental symmetry tests. Nat Commun. 8, 681 (2017)

16. Capra, A. \& ALPHA Collaboration. Lifetime of magnetically trapped antihydrogen in ALPHA. Hyperfine Interact. 240, 9 (2019).

17. Ahmadi, M. et al. Observation of the hyperfine spectrum of antihydrogen. Nature $\mathbf{5 4 8}$ 66-69 (2017); erratum 553, 530 (2018).

18. Michan, J. M., Polovy, G., Madison, K. W., Fujiwara, M. C. \& Momose, T. Narrowband solid state VUV coherent source for laser cooling of antihydrogen. Hyperfine Interact. 235 29-36 (2015).

19. Andresen, G. B. et al. Trapped antihydrogen. Nature 468, 673-676 (2010).

20. Andresen, G. B. et al. Confinement of antihydrogen for 1,000 seconds. Nat. Phys. 7, 558-564 (2011).

21. Ahmadi, M. et al. Observation of the $1 S-2 S$ transition in trapped antihydrogen. Nature 541, 506-510 (2017).

22. Ahmadi, M. et al. Enhanced control and reproducibility of non-neutral plasmas. Phys. Rev. Lett. 120, 025001 (2018).

23. Maury, S. The antiproton decelerator: AD. Hyperfine Interact. 109, 43-52 (1997).

24. Murphy, T. J. \& Surko, C. M. Positron trapping in an electrostatic well by inelastic collisions with nitrogen molecules. Phys. Rev. A 46, 5696-5705 (1992).

25. Surko, C. M., Greaves, R. G. \& Charlton, M. Stored positrons for antihydrogen production. Hyperfine Interact. 109, 181-188 (1997).

26. Luiten, O. J. et al. Lyman-a spectroscopy of magnetically trapped atomic hydrogen. Phys. Rev. Lett. 70, 544-547 (1993).

27. Eikema, K. S. E., Walz, J. \& Hänsch, T. W. Continuous coherent Lyman-a excitation of atomic hydrogen. Phys. Rev. Lett. 86, 5679-5682 (2001).

28. Gabrielse, G. et al. Lyman-a source for laser cooling antihydrogen. Opt. Lett. 43, 2905-2908 (2018)
29. Stracka, S. Real-time detection of antihydrogen annihilations and applications to spectroscopy. EPJ Web Conf. 71, 00126 (2014)

30. Donnan, P. H., Fujiwara, M. C. \& Robicheaux, F. A proposal for laser cooling antihydrogen atoms. J. Phys. B 46, 025302 (2013).

31. Pohl, R. et al. The size of the proton. Nature 466, 213-216 (2010).

32. Beyer, A. et al. The Rydberg constant and proton size from atomic hydrogen. Science 358, 79-85 (2017)

33. Fleurbaey, $\mathrm{H}$. et al. New measurement of the transition frequency of hydrogen: contribution to the proton charge radius puzzle. Phys. Rev. Lett. 120, 183001 (2018)

34. The ALPHA Collaboration \& Charman, A. E. Description and first application of a new technique to measure the gravitational mass of antihydrogen. Nat. Commun. 4, 1785 (2013).

Publisher's note Springer Nature remains neutral with regard to jurisdictional claims in published maps and institutional affiliations.

cc) (1)

Open Access This article is licensed under a Creative Commons Attribution 4.0 International License, which permits use, sharing, adaptation, distribution and reproduction in any medium or format, as long as you give appropriate credit to the original author(s) and the source, provide a link to the Creative Commons license, and indicate if changes were made. The images or other third party material in this article are included in the article's Creative Commons license, unless indicated otherwise in a credit line to the material. If material is not included in the article's Creative Commons license and your intended use is not permitted by statutory regulation or exceeds the permitted use, you will need to obtain permission directly from the copyright holder. To view a copy of this license, visit http://creativecommons.org/licenses/by/4.0/.

(c) The Author(s) 2020

The ALPHA Collaboration

M. Ahmadi', B. X. R. Alves ${ }^{2}$, C. J. Baker ${ }^{3}$, W. Bertsche ${ }^{4,5}$, A. Capra ${ }^{6}$, C. Carruth ${ }^{7}$, C. L. Cesar ${ }^{8}$, M. Charlton ${ }^{3}$, S. Cohen ${ }^{9}$, R. Collister ${ }^{6}$, S. Eriksson ${ }^{3}$, A. Evans ${ }^{10}$, N. Evetts ${ }^{11}$, J. Fajans ${ }^{7}$, T. Friesen $^{2,10}$, M. C. Fujiwara ${ }^{6 凶}$, D. R. Gill ${ }^{6}$, P. Granum ${ }^{2}$, J. S. Hangst ${ }^{2}$, W. N. Hardy ${ }^{11}$, M. E. Hayden $^{12}$, E. D. Hunter ${ }^{7}$, C. A. Isaac ${ }^{3}$, M. A. Johnson ${ }^{4,5}$, J. M. Jones ${ }^{3}$, S. A. Jones ${ }^{2,3}$, S. Jonsell ${ }^{13}$, A. Khramov ${ }^{6,11}$, P. Knapp ${ }^{3}$, L. Kurchaninov ${ }^{6}$, N. Madsen ${ }^{3}$, D. Maxwell ${ }^{3}$, J. T. K. McKenna $^{2,6}$, S. Menary ${ }^{14}$, J. M. Michan ${ }^{6,15}$, T. Momose ${ }^{11,16}{ }^{凶}$, J. J. Munich ${ }^{12}$, K. Olchanski ${ }^{6}$, A. Olin $^{6,17}$, P. Pusa', C. Ø. Rasmussen ${ }^{2}$, F. Robicheaux ${ }^{18}$, R. L. Sacramento ${ }^{8}$, M. Sameed ${ }^{4}$, E. Sarid $^{19}$, D. M. Silveira ${ }^{8}$, C. So ${ }^{6,10}$, D. M. Starko ${ }^{14}$, G. Stutter ${ }^{2}$, T. D. Tharp ${ }^{19,20}$, R. I.

Thompson $^{6,10}$, D. P. van der Werf ${ }^{3,21} \&$ J. S. Wurtele ${ }^{7}$

${ }^{1}$ Department of Physics, University of Liverpool, Liverpool, UK. ${ }^{2}$ Department of Physics and Astronomy, Aarhus University, Aarhus, Denmark. ${ }^{3}$ Department of Physics, College of Science, Swansea University, Swansea, UK. ${ }^{4}$ School of Physics and Astronomy, University of Manchester, Manchester, UK. ${ }^{5}$ Cockcroft Institute, Warrington, UK. ${ }^{6}$ TRIUMF, Vancouver, British Columbia, Canada. ${ }^{7}$ Department of Physics, University of California at Berkeley, Berkeley, CA, USA. ${ }^{8}$ Instituto de Fisica, Universidade Federal do Rio de Janeiro, Rio de Janeiro, Brazil. ' ${ }^{9}$ pepartment of Physics, Ben-Gurion University of the Negev, Beer-Sheva, Israel. ${ }^{10}$ Department of Physics and Astronomy, University of Calgary, Calgary, Alberta, Canada. "Department of Physics and Astronomy, University of British Columbia, Vancouver, British Columbia, Canada. ${ }^{12}$ Department of Physics, Simon Fraser University, Burnaby, British Columbia, Canada. ${ }^{13}$ Department of Physics, Stockholm University, Stockholm, Sweden. ${ }^{14}$ Department of Physics and Astronomy, York University, Toronto, Ontario, Canada. ${ }^{15}$ École Polytechnique Fédérale de Lausanne (EPFL), Swiss Plasma Center (SPC), Lausanne, Switzerland. ${ }^{16}$ Department of Chemistry, University of British Columbia, Vancouver, British Columbia, Canada. ${ }^{17}$ Department of Physics and Astronomy, University of Victoria, Victoria, British Columbia, Canada. ${ }^{18}$ Department of Physics and Astronomy, Purdue University, West Lafayette, IN, USA. ${ }^{19}$ Soreq NRC, Yavne, Israel. ${ }^{20}$ Physics Department, Marquette University, Milwaukee, WI, USA. ${ }^{21}$ IRFU, CEA/Saclay, Gif-sur-Yvette, France. ${ }^{\bowtie}$ e-mail: Makoto.Fujiwara@ triumf.ca; jeffrey.hangst@cern.ch; momose@chem.ubc.ca 


\section{Methods}

\section{Transition-frequency determination}

The observed 1S-2P transition spectra have asymmetric shapes with a low-frequency tail caused by Zeeman shifts in the inhomogeneousmagnetic-field regions away from the centre of the ALPHA-2 trap. As a result, the apparent peak of the observed spectrum is shifted to a slightly lower frequency with respect to the resonance transition frequency $f_{\text {res }}$, which is defined for atoms in resonance at the magneticfield minimum of the trap. This offset is relatively small (of the order of $50 \mathrm{MHz}$ ). Nonetheless, we performed extensive analysis to understand the effects of this asymmetry on our transition-frequency determination. The details of the analysis follow.

A detailed simulation was used to model the motion of trapped antihydrogen atoms in the ALPHA-2 trap, as well as their interaction with pulsed laser radiation. Aspects of our simulation have been validated in previous studies (for example, refs. ${ }^{10,11,19-24}$ ). To determine the resonance transition frequency, we first simulated lineshapes for the transitions from the two trappable $1 \mathrm{~S}$ hyperfine states to the $2 \mathrm{P}_{\mathrm{c}}$ and $2 \mathrm{P}_{\mathrm{f}}$ excited states (that is, for four transitions: $1 \mathrm{~S}_{\mathrm{c}} \rightarrow 2 \mathrm{P}_{\mathrm{c}^{+}}, 1 \mathrm{~S}_{\mathrm{c}} \rightarrow 2 \mathrm{P}_{\mathrm{f}+}$, $1 \mathrm{~S}_{\mathrm{d}} \rightarrow 2 \mathrm{P}_{\mathrm{c}^{-}}$and $1 \mathrm{~S}_{\mathrm{d}} \rightarrow 2 \mathrm{P}_{\mathrm{f}-}$ ). We then fitted each component with an asymmetric lineshape function, referred to as GE. GE is a Gaussian spliced to an exponential low frequency tail, where the derivative of the crossover point is required to be continuous. GE has four parameters: the peak frequency $\left(f_{\text {peak }}\right)$ and the width $(W)$ of the Gaussian, the crossover point frequency $\left(f_{\mathrm{x}}\right)$ and the overall amplitude $(A)$. From the fit, we determined the simulated lineshape parameters $f_{\text {peak }}(\operatorname{sim}), W(\operatorname{sim}), f_{\mathrm{x}}(\operatorname{sim})$ and $A(\operatorname{sim})$ for each transition. In addition, we derived the peak frequency offset $\Delta f$, defined as $\Delta f=f_{\text {peak }}(\operatorname{sim})-f_{\text {res }}($ th $)$, where $f_{\text {res }}($ th $)$ is the expected theoretical resonance frequency for hydrogen in the magnetic field $B$.

The experimentally observed spectra were then fitted with $G E$ lineshapes. A sum of two GEs was used to fit singly spin-polarized samples, where only $f_{\text {peak }}$ and a single normalization factor were used as the fitting parameters, whereas the rest of the parameters (that is, the $W$ and $f_{\mathrm{x}}$ values of each GE, the spacing of $f_{\text {peak }}$ between two GEs, and the ratio of the amplitudes $A$ of two GEs) were fixed to the corresponding simulated values. For doubly spin-polarized samples, the experimental spectra were fitted with a single GE lineshape. In these fits, $W$ and $f_{\mathrm{x}}$ were fixed using a fit to the simulated spectrum in which an estimated $5 \%$ contamination of the $1 \mathrm{~S}_{\mathrm{c}}$ component was assumed. The experimental transition frequency is given by $f_{\text {res }}(\exp )=f_{\text {peak }}(\exp )-\Delta f$, where $f_{\text {peak }}(\exp )$ is the peak frequency of the experimental data obtained by the fit. Here $\Delta f$ corrects for the asymmetric lineshape as described earlier. The red lines (labelled as 'Model 1') in Fig. 3 show the results of these fits using standard simulations. We note that the transition to the $2 \mathrm{P}_{\mathrm{e}}$ state is allowed when the laser polarization is not perfectly perpendicular to the $B$ field. This could arise from the slight angle between the laser and the magnetic field (maximum $4^{\circ}$ at the edge of our trap) or from a possible nonlinear component in the polarization of the 121-nm light (expected to be of the order of $10 \%$ or less). The frequency of the $1 S-2 \mathrm{P}_{\mathrm{e}}$ transition is well separated from that of the $1 \mathrm{~S}-2 \mathrm{P}_{\mathrm{c}}$ transition (by about $-3.5 \mathrm{GHz}$ ), and its predicted intensity is very small (less than a few per cent of that for the $1 \mathrm{~S}-2 \mathrm{P}_{\mathrm{c}}$ transition), hence it was ignored in the analysis.

\section{Transition-frequency uncertainties}

Extensive studies were performed to quantify the uncertainties in our frequency determination. The standard simulated spectra reproduce the observed lineshape reasonably well without any fine-tuning (Extended Data Fig. 1). The sensitivity of the obtained resonance frequency $f_{\text {res }}(\exp )$ to the input parameters in the simulation was studied by varying these input parameters and repeating the same analysis.

The standard input to the simulation and the range of the parameters studied (given in parentheses), were as follows. Laser pulse energies, $500 \mathrm{pJ}(350 \mathrm{pJ}, 800 \mathrm{pJ})$; laser line linewidth, $65 \mathrm{MHz}(50 \mathrm{MHz}, 80 \mathrm{MHZ})$; relative magnitude of the laser sideband (present at $+90 \mathrm{MHz}$ with respect to the main band owing to multimode lasing in the $730-\mathrm{nm}$ amplification cavity), $10 \%(0 \%, 25 \%)$; radial position displacement of the laser beam: $0 \mathrm{~mm}(0 \mathrm{~mm}, 3 \mathrm{~mm})$; initial quantum state of antihydrogen at formation: $n=30(1,30)$; initial diameter of the cloud of antihydrogen: $0.45 \mathrm{~mm}(0.45 \mathrm{~mm}, 0.90 \mathrm{~mm})$; temperature of antihydrogen at formation (before trapping): $15 \mathrm{~K}(1 \mathrm{~K}, 15 \mathrm{~K})$.

An alternative fitting method was also used to study the robustness of our procedure. Here, the lineshape function GE was fitted to the data without using constraints from the simulated spectrum. From the fit, $f_{\text {peak }}(\exp )$ was extracted for each transition, and the experimental resonance frequency was determined as $f_{\text {res }}(\exp )=f_{\text {peak }}(\exp )-\Delta f$, where the offset $\Delta f$ from the standard simulation was assumed. The lineshapes given by these fits are shown by blue lines (labelled as 'Model 2') in Fig. 3.

The results of the analyses using the simulations with varied input parameters, as well as alternative fitting models, are given by red lines in Extended Data Fig. 1, which illustrates that the dependence on the details of the fitting procedure is small. The variations of the extracted frequency $f_{\text {res }}(\exp )$ in these studies (both with different simulation inputs and different fitting methods) were generally within the statistical uncertainties of these fits. We took the largest deviations in $f_{\text {res }}(\exp )$ among these studies as a measure of the fitting-model dependence (Table 3).

It should be noted that our evaluation of the fitting-model dependence systematics relies on the GE model being a reasonable representation of the simulated data. This agreement is qualitatively illustrated in Extended Data Fig. 1. Quantitatively, for the simulations with the standard input parameters, the $\chi^{2}$ per degree of freedom (DOF) ranges from 1.2 to 2.5 (with an average of 1.8 ), where DOF $=8$. When the input parameters are varied in the fits to the data, the $\chi^{2}$ per DOF ranges from 1.0 to 3.9 , with an average of 2.1 . The simulation statistics were roughly a factor of 2-4 greater than the data; hence, the uncertainties arising from our analytical model of the simulation lineshape are small.

The sources of uncertainty in the transition frequencies can be summarized as follows (we note that the frequency uncertainties at $730 \mathrm{~nm}$ should be multiplied by a factor of 6 to give those at $121 \mathrm{~nm}$ ): (a) Wavemeter drift: this is due to temperature-induced drift of the wavemeter readings, which was estimated from offline studies to be about $20 \mathrm{MHz} \mathrm{K}^{-1}$ at $730 \mathrm{~nm}$. Given the recorded temperature variation of $\pm 0.25 \mathrm{~K}$, we assigned an error of $\pm 5 \mathrm{MHz}$ at $730 \mathrm{~nm}$. We note that a temperature drift during our 2-h measurements would result in a broadening of the observed linewidth. This effect would be also taken into account partly by the fitting-model uncertainty discussed above. Therefore, there is a possibly of partial double counting, but we conservatively list both effects separately. (b) Wavemeter offset: an offset of the He-Ne laser calibration source, estimated to be $\pm 3 \mathrm{MHz}$ at $730 \mathrm{~nm}$ by offline calibration. (c) 730-nm cavity resonance-frequency correction: the frequency of the generated $730-\mathrm{nm}$ pulse was measured to be shifted from that of the continuous-wave 730-nm seed laser. This shift of about $10 \mathrm{MHz}$ at $730 \mathrm{~nm}$ was regularly monitored, and was corrected for in our frequency determination. We conservatively assign an error of $10 / \sqrt{\mathbf{1 2}}=3 \mathrm{MHz}$ to this correction at $730 \mathrm{~nm}$ (the standard deviation of a uniform distribution with a width of $10 \mathrm{MHz}$ ). (d) Residual $\mathrm{SS}_{\mathrm{c}}$ state contamination: our earlier studies with shorter running times $^{11,22}$ indicate there is a residual population of the order of $5 \%$ of the $1 S_{c}$ state after the microwave-driven clearing procedure, which was corrected for in the analysis above. We estimate the error in this correction by analysing the data assuming no residual $1 \mathrm{~S}_{\mathrm{c}}$ population. We take $68 \%$ of the differences between the two analysis results $(33.5 \mathrm{MHz}$ and $24 \mathrm{MHz}$ for the $2 \mathrm{P}_{\mathrm{c}}$ and $2 \mathrm{P}_{\mathrm{f}}$ transitions, respectively) as $1 \sigma$ uncertainties in the correction. (e) Magnetic field: the field at the magnetic minimum of the ALPHA-2 trap was measured in situ using the electron cyclotron resonance (ECR) method ${ }^{35}$. A conservative uncertainty of $10 \mathrm{MHz}$ in the ECR measurement gives a $B$ field error of $3.6 \times 10^{-4} \mathrm{~T}$, which in turn gives frequency errors of $5 \mathrm{MHz}$ and $8 \mathrm{MHz}$ for the $1 \mathrm{~S}-2 \mathrm{P}_{\mathrm{c}}$ and $1 \mathrm{~S}-2 \mathrm{P}_{\mathrm{f}}$ transitions, respectively, at $1 \mathrm{~T}$. We take these values as a 
measure of the uncertainty due both to the absolute value and to the run-to-run stability of the $B$ field. We note that the frequency uncertainty in the $1 \mathrm{~S}-2 \mathrm{~S}$ transition due to $B$-field variations is negligible for our purposes $^{11}$. (f) Statistical uncertainties of the fit: these represent statistical uncertainties in the fit both from the experimental data and from the simulations. (g) Model uncertainties: described above.

The total errors for each transition are given by the quadratic sum of errors (a)-(g). Care must be taken when taking an average or a difference of the transition frequencies. Here we assume that error (b), the wavemeter offset, introduces a common offset to all the data series. The other errors are assumed to be uncorrelated across the dataset. The resulting combined uncertainty for the transition frequencies of antihydrogen is $39 \mathrm{MHz}$ or $16 \mathrm{ppb}$ (Fig. 4, average value). We expect that virtually all of the uncertainties can be considerably reduced in the near future owing to increased statistics and improved control of the systematics.

\section{Determination of the fine-structure splitting and the Lamb shift of antihydrogen}

To analyse the Zeeman-shifted energy levels of antihydrogen in the $2 \mathrm{P}$ state, we used the following Hamiltonian for the 2P state, which includes the field-free Hamiltonian $\left(\hat{H}_{0}\right)$, the fine-structure Hamiltonian $\left(\hat{H}_{\mathrm{fs}}\right)$, the Zeeman Hamiltonian $\left(\hat{H}_{\mathrm{Z}}\right)$ and the hyperfine-structure Hamiltonian $\left(\hat{H}_{\mathrm{hf}}\right)$ :

$$
\begin{gathered}
\hat{H}=\hat{H}_{0}+\hat{H}_{\mathrm{fs}}+\hat{H}_{\mathrm{Z}}+\hat{H}_{\mathrm{hf}} \\
\hat{H}_{\mathrm{fs}}=\frac{2}{3} \overline{\mathcal{E}}_{\mathrm{fs}}\left(\frac{1}{\hbar^{2}} \mathbf{L}_{\bar{e}} \cdot \mathbf{S}_{\bar{e}}+1\right) \\
\hat{H}_{\mathrm{Z}}=-\frac{2 \mu_{\bar{e}}(2 \mathrm{P})}{\hbar} \mathbf{S}_{\bar{e}} \cdot \mathbf{B}-\frac{2 \mu_{\bar{p}}}{\hbar} \mathbf{I}_{\bar{p}} \cdot \mathbf{B}+\frac{\tilde{\mu}_{\bar{B}}}{\hbar} \mathbf{L}_{\bar{e}} \cdot \mathbf{B} \\
\hat{H}_{\mathrm{hf}}=\frac{\bar{C}_{l L}}{\hbar^{2}} \mathbf{I}_{\bar{p}} \cdot \mathbf{L}_{\bar{e}}+\frac{\bar{C}_{I S}}{\hbar^{2}}\left[\mathbf{I}_{\bar{p}} \cdot \mathbf{S}_{\bar{e}}-3\left(\mathbf{I}_{\bar{p}} \cdot \mathbf{r}\right)\left(\mathbf{S}_{\bar{e}} \cdot \mathbf{r}\right)\right]
\end{gathered}
$$

Here, $\mathbf{L}_{\bar{e}}$ is the orbital angular momentum of the positron, $\mathbf{S}_{\bar{e}}$ is the spin angular momentum of the positron, $\mathbf{I}_{\bar{p}}$ is the nuclear spin angular momentum of the antiproton and $\mathbf{r}$ is the position vector of the positron. $\overline{\mathcal{E}}_{\mathrm{fs}}$ is the fine-structure splitting of antihydrogen at zero field. The magnetic moments of the positron and antiproton are given by $\mu_{\bar{e}}(2 \mathrm{P})=\frac{\left|\bar{g}_{\mathrm{s}}\right|}{2} \frac{|\bar{e}| \hbar}{2 m_{\bar{e}}}\left(1-\frac{\alpha^{2}}{10}\right)$ and $\mu_{\bar{p}}=-\frac{\left|\bar{g}_{\mathrm{p}}\right|}{2} \frac{|\bar{e}| \hbar}{2 m_{\bar{p}}}$ where $\bar{g}_{\mathrm{s}}$ and $\bar{g}_{\mathrm{p}}$ are the positron spin and antiproton $g$-factors, respectively, $\bar{e}$ and $m_{\bar{e}}$ are the charge and mass of the positron, correspondingly, and $\alpha$ is the finestructure constant. The last term of equation (4) is the Zeeman interaction due to the orbital angular momentum of the positron with magnetic moment of $\widetilde{\mu}_{\bar{B}}=-\left(1-\frac{m_{\bar{e}}}{m_{\bar{p}}}\right) \frac{|\bar{e}| \hbar}{2 m_{\bar{e}}}$, where $m_{\bar{p}}$ is the mass of the antiproton. $\bar{C}_{I L}$ is the hyperfine-coupling constant due to the antiproton spin and the orbital angular momentum of the positron, and $\bar{C}_{I S}$ is the hyperfine interaction due to the magnetic dipole-dipole interaction.

For the analysis of the classic Lamb shift $\left(\overline{\mathcal{E}}_{\text {Lamb }}\right)$ and the fine-structure $\left(\overline{\mathcal{E}}_{\mathrm{fs}}\right)$ parameters of antihydrogen, we assumed that the absolute values of the three magnetic moments $\left(\mu_{\bar{e}}, \mu_{\bar{p}}\right.$ and $\left.\tilde{\mu}_{\bar{B}}\right)$ are the same as those of hydrogen. Previous measurements of the basic properties of antiparticles are consistent with this assumption. The hyperfine-coupling constants are also assumed to be those of hydrogen ${ }^{38}, \bar{C}_{I L}=22.2 \mathrm{MHz}$ and $\bar{C}_{I S}=-22.2 \mathrm{MHz}$.

Our measurements determine the energy levels, with respect to the $1 \mathrm{~S}$ ground state, of two of the Zeeman sublevels in the $n=2$ positronic manifold of antihydrogen at a magnetic field of $1.0329 \mathrm{~T}$. Specifically, the $2 \mathrm{P}_{\mathrm{f}}$ state belongs to the $2 \mathrm{P}_{1 / 2}$ manifold, and the $2 \mathrm{P}_{\mathrm{c}}$ state belongs to the $2 \mathrm{P}_{3 / 2}$ manifold (see Fig. 1 ). We combine these results with our previous measurement of the $1 S_{d}-2 S_{d}$ transition ${ }^{7}$ and assume the validity of the standard Zeeman and hyperfine interactions to derive the finestructure splitting $\overline{\mathcal{E}}_{\mathrm{fS}}$ (that is, the energy difference between $2 \mathrm{P}_{1 / 2}$ and $2 \mathrm{P}_{3 / 2}$ ), and the classic Lamb shift $\overline{\mathcal{E}}_{\text {Lamb }}$ (that is, the energy difference between $2 \mathrm{~S}_{1 / 2}$ and $2 \mathrm{P}_{1 / 2}$ ), both defined at zero field.

Taking into account the hyperfine splitting, we find the energy separation between the $2 \mathrm{P}_{\mathrm{c}^{-}}$and $2 \mathrm{P}_{\mathrm{f}-}$ levels at $1.0329 \mathrm{~T}$ to be $14.945 \pm 0.0975 \mathrm{GHz}$, from the difference of the weighted average values of the observed transition frequencies. Furthermore, we obtain the separation between the $2 \mathrm{~S}_{\mathrm{d}}$ and $2 \mathrm{P}_{\mathrm{c}-}$ levels to be $\Delta E\left(2 \mathrm{~S}, 2 \mathrm{P}_{\mathrm{c}}\right)=9,832 \pm 49 \mathrm{MHz}$, and that between the $2 \mathrm{~S}_{\mathrm{d}}$ and $2 \mathrm{P}_{\mathrm{f}-}$ levels to be $\Delta E\left(2 \mathrm{~S}, 2 \mathrm{P}_{\mathrm{f}}\right)=24,778 \pm 60 \mathrm{MHz}$, in the same field. The sum and the difference of the two quantities, $\Delta E\left(2 \mathrm{~S}, 2 \mathrm{P}_{c}\right)$ and $\Delta E\left(2 \mathrm{~S}, 2 \mathrm{P}_{\mathrm{f}}\right)$, can be expressed by the following equations, which are based on the standard Hamiltonian of the hydrogen atom in a magnetic field $B$ (refs. ${ }^{36,37}$ ). We neglect terms that contribute less than $1 \mathrm{MHz}$.

$$
\begin{aligned}
& \Delta E\left(2 \mathrm{~S}, 2 \mathrm{P}_{\mathrm{f}}\right)-\Delta E\left(2 \mathrm{~S}, 2 \mathrm{P}_{\mathrm{c}}\right)= \\
& 2 E_{1}(-B)+\frac{1}{2} \bar{C}_{I L} \cos (2 \sigma)+\frac{1}{10}[\cos (2 \sigma)-3 \sqrt{2} \sin (2 \sigma)] \bar{C}_{I S} \\
& \Delta E\left(2 \mathrm{~S}, 2 \mathrm{P}_{\mathrm{f}}\right)+\Delta E\left(2 \mathrm{~S}, 2 \mathrm{P}_{\mathrm{c}}\right)= \\
& 2 \overline{\mathcal{E}}_{\text {Lamb }}-2 \overline{\mathcal{E}}_{\mathrm{fs}}+\frac{1}{2} \bar{C}_{I L}-\frac{3}{10} \bar{C}_{I S}+\frac{1}{2} \overline{\mathcal{E}}_{\mathrm{hf}}(2 \mathrm{~S})-\left[2 \mu_{\bar{e}}(2 \mathrm{P})+\tilde{\mu}_{\bar{B}}\right] B
\end{aligned}
$$

where

$$
E_{1}(-B)=\sqrt{\left\{\frac{1}{6} \overline{\mathcal{E}}_{\mathrm{fs}}-B\left[\mu_{\bar{e}}(2 \mathrm{P})+\frac{1}{2} \widetilde{\mu}_{\bar{B}}\right]\right\}^{2}+\frac{2}{9} \overline{\mathcal{E}}_{\mathrm{fs}}^{2}}
$$

and

$$
\tan \sigma=\frac{-\overline{\mathcal{E}}_{\mathrm{fs}}+\left[6 \mu_{\bar{e}}(2 \mathrm{P})+3 \tilde{\mu}_{\bar{B}}\right] B+6 E_{1}(-B)}{2 \sqrt{2} \overline{\mathcal{E}}_{\mathrm{fs}}}
$$

Here, $\overline{\mathcal{E}}_{\mathrm{hf}}(2 \mathrm{~S})$ is the hyperfine splitting in the $2 \mathrm{~S}$ state at zero field.

Finally, using the CODATA 2014 values of the fundamental constants for the hydrogen atom ${ }^{39}$, the fine-structure splitting $\overline{\mathcal{E}}_{\text {hf }}$ and the classic Lamb shift $\overline{\mathcal{E}}_{\text {Lamb }}$ of the antihydrogen atom are determined by numerically solving equations (5) and (6) with the measured energy-level differences given in Table 2 as input.

\section{Hydrogen transition frequencies in a magnetic field}

From zero-field measurements in hydrogen for the $1 \mathrm{~S}_{1 / 2}-2 \mathrm{~S}_{1 / 2}\left(\right.$ ref. $^{40}$ ), $2 \mathrm{~S}_{1 / 2}-2 \mathrm{P}_{1 / 2}\left(\right.$ ref. $^{41}$ ) and $2 \mathrm{P}_{1 / 2}-2 \mathrm{P}_{3 / 2}\left(\right.$ ref. $^{42}$ ) transitions, we obtain hyperfine centroid frequencies of

$$
\begin{aligned}
& 1 \mathrm{~S}-2 \mathrm{P}_{1 / 2} \text { transition: } 2,466,060,355 \mathrm{MHz} \\
& 1 \mathrm{~S}-2 \mathrm{P}_{3 / 2} \text { transition: } 2,466,071,324 \mathrm{MHz}
\end{aligned}
$$

The transition frequencies at $1.0329 \mathrm{~T}$ (Table 2) are calculated by evaluating corrections assuming the standard Zeeman, fine-structure and hyperfine interactions in a magnetic field ${ }^{36,37}$ and using the current CODATA values of the fundamental constants ${ }^{39}$. The precision of our calculations is better than $1 \mathrm{MHz}$.

In comparing the hydrogen values with the measured antihydrogen frequencies in Table 2 and Fig. 4 , the value of the magnetic field was assumed to be exact for the hydrogen case.

\section{Data availability}

The datasets generated and/or analysed during the current study are available from J.S.H. on reasonable request.

\footnotetext{
35. Amole, C. et al. In situ electromagnetic field diagnostics with an electron plasma in a Penning-Malmberg trap. New J. Phys. 16, 013037 (2014).

36. Rasmussen, C. Ø., Madsen, N. \& Robicheaux, F. Aspects of 1S-2S spectroscopy of trapped antihydrogen atoms. J. Phys. B 50, 184002 (2017); corrigendum 51099501 (2018).
} 
37. Melezhik, V. S. \& Schmelcher, P. Quantum energy flow in atomic ions moving in magnetic fields. Phys. Rev. Lett. 84, 1870-1873 (2000).

38. Kramida, A. E. A critical compilation of experimental data on spectral lines and energy levels of hydrogen, deuterium, and tritium. At. Data Nucl. Data Tables 96, 586-644 (2010); erratum 126, 295-298 (2019).

39. Mohr, P. J., Newell, D. B. \& Taylor, B. N. CODATA recommended values of the fundamental physical constants: 2014. Rev. Mod. Phys. 88, 035009 (2016).

40. Parthey, C. G. et al. Improved measurement of the hydrogen $1 \mathrm{~S}-2 \mathrm{~S}$ transition frequency. Phys. Rev. Lett. 107, 203001 (2011).

41. Lundeen, S. R. \& Pipkin, F. M. Measurement of the Lamb shift in hydrogen $n=2$. Phys. Rev. Lett. 46, 232-235 (1981)

42. Hagley, E. W. \& Pipkin, F. M. Separated oscillatory field measurement of hydrogen $2 \mathrm{~S}_{1 / 2}-2 \mathrm{P}_{3 / 2}$ fine structure interval. Phys. Rev. Lett. 72, 1172-1175 (1994).

Acknowledgements This work was supported by: the European Research Council through its Advanced Grant programme (J.S.H.); CNPq, FAPERJ, RENAFAE (Brazil); NSERC, ALPHA-g/ CRUCS CFI, NRC/TRIUMF, EHPDS/EHDRS (Canada); FNU (Nice Centre), Carlsberg Foundation (Denmark); ISF (Israel); STFC, EPSRC, the Royal Society and the Leverhulme Trust (UK); DOE, NSF (USA); and VR (Sweden). We are grateful for the efforts of the CERN AD team, without which these experiments could not have taken place. We thank P. Djuricanin (University of British Columbia) for extensive help with the laser system and calibrations. We thank J. Tonoli and the CERN staff, as well as T. Mittertreiner and the UBC staff, for extensive, time-critical help with machining and electrical works. We thank the staff of the

Superconducting Magnet Division at Brookhaven National Laboratory for collaboration and for the fabrication of the trapping magnets. We thank C. Marshall (TRIUMF) for work on the ALPHA-2 cryostat. We thank F. Besenbacher (Aarhus) for timely support in procuring the
ALPHA-2 external solenoid. We thank T. Miller (Ohio) for advice on the initial development of the pulsed laser system. We thank A. Kostelecky and G. Drake for discussions on the theoretical aspects of this work.

Author contributions This study was based on data collected using the ALPHA-2 antihydrogen trapping apparatus, designed and constructed by the ALPHA Collaboration using methods developed by the entire collaboration. The entire collaboration participated in the operation of the apparatus and the data-taking activities. Pulsed Lyman-a spectroscopy was first proposed by M.C.F. and developed further by T.M., F.R., J.M.M., R.C., A.E., A.K. and M.C.F. The original laser was designed by T.M. and tested by J.M.M. and T.M. The laser system at CERN was implemented, commissioned and operated by J.M.M., R.C., A.E., A.K. and T.M. The simulation program for laser interaction with magnetically trapped atoms was developed by F.R. The microwave techniques and ECR magnetometry were developed by T.F., M.E.H. and W.N.H. The positron accumulator is the responsibility of C.J.B., M.C., M.S., C.A.I. and D.P.v.d.W. The annihilation detector system was developed by A.C., M.C.F., D.R.G., L.K., J.T.K.M., S.M., K.O., A.O. and P.P. Detailed analysis of the antiproton annihilation detector data was done by J.T.K.M., A.E. and A.O. The frequency determination and the evaluation of its uncertainty was performed by T.M., M.C.F., A.K., A.E., R.C., R.I.T. and A.O. The manuscript was written by T.M., M.C.F. and J.S.H., with significant input from A.K., R.C., A.E., A.O., J.T.K.M., M.E.H., N.M., C.Ø.R. and F.R. The manuscript was edited and improved by the entire collaboration.

Competing interests The authors declare no competing interests.

Additional information

Correspondence and requests for materials should be addressed to M.C.F., J.S.H. or T.M. Reprints and permissions information is available at http://www.nature.com/reprints. 


\section{Article}
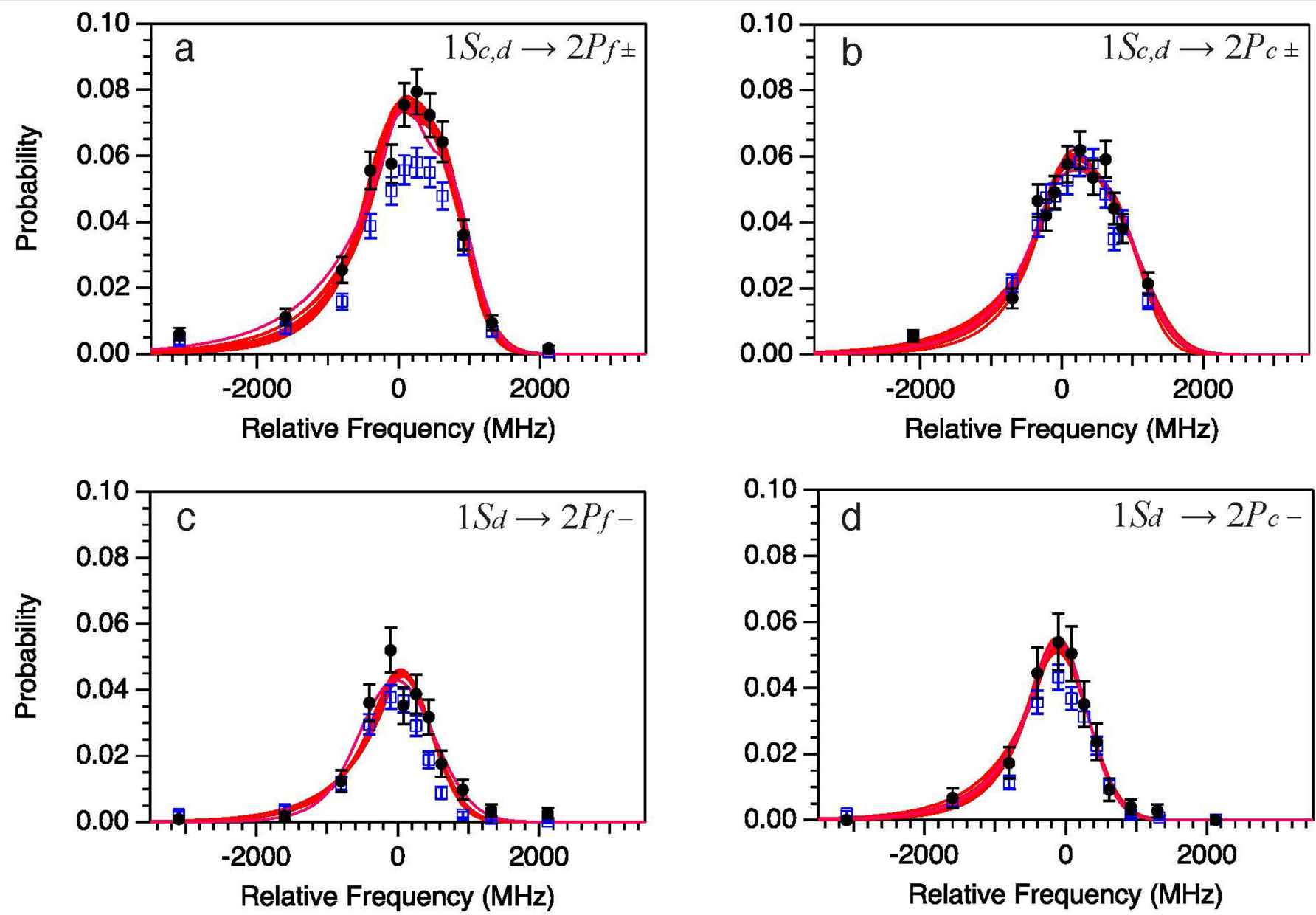

Extended Data Fig. 1 | Determination of transition frequencies. a-d, For each series, the experimental data (filled black circles with error bars) are plotted with fits of various models (red lines) discussed in Methods. The experimental data are normalized to the total number of the detected antihydrogen atoms and a laser power of $5 \mathrm{nW}$. Also shown are the results of standard simulations (open blue squares with error bars), similarly normalized to the total number of

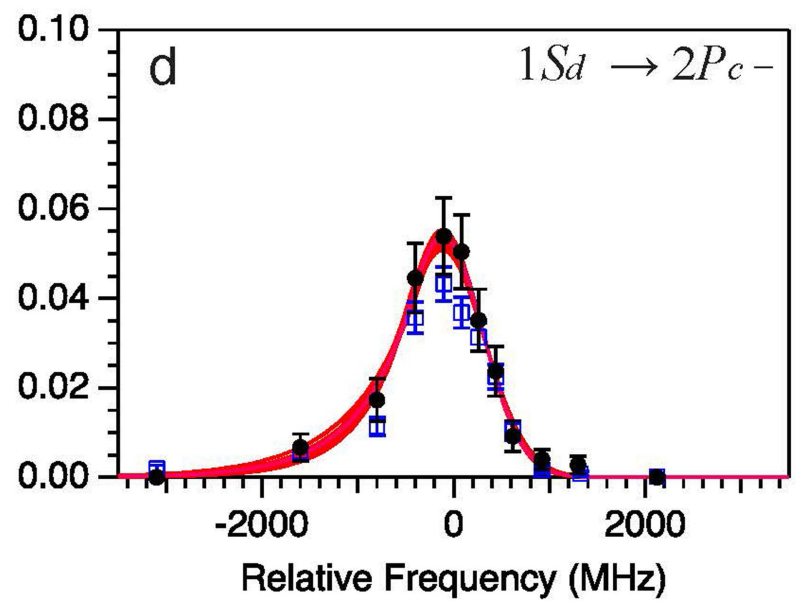

simulated atoms, illustrating the degree of agreement between the data and the simulations, without any tuning parameters. Some discrepancies in the amplitudes can be observed, which may point to errors in our laser power estimates. We note that because our frequency-fitting procedure allows variations in the relative amplitudes, the fits are largely insensitive to the amplitude differences (Methods). Error bars represent $1 \sigma$. 